\title{
PERTINENCIA CURRICULAR DE LA CARRERA DE INFORMÁTICA ADMINISTRATIVA DE URACCAN, RECINTO BILWI
}

\author{
Ivonne McLean Diesen ${ }^{[1]}$ \\ Sandra Hooker Hodgson ${ }^{[2]}$
}

\section{Resumen}

Se ha investigado la pertinencia del currículo de la carrera de Informática Administrativa de la Universidad de las Regiones Autónomas de la Costa Caribe de Nicaragua (URACCAN) Recinto Bilwi. El estudio fue cualitativo, evaluativo y de corte transversal, el objetivo fue el estudio de la pertinencia del currículo en la carrera de Informática Administrativa, de dicho Recinto Universitario. Las técnicas de recolección de información fueron: entrevistas, grupo focal y revisión de documentos. Para el procesamiento de la información se utilizó la tabla matricial para analizar críticamente estas informaciones.

El hallazgo principal del estudio ha reflejado que el currículo de la carrera de Informática Administrativa, tiene varios aspectos positivos. Sin embargo, este no es pertinente, ya que la evaluación de la dimensión externa es pertinente, no así la interna y la administrativa. Como las tres dimensiones deben estar íntimamente interrelacionadas y al no haber congruencia en una de estas dimensiones afecta la pertinencia de todo el currículo de la carrera. Esta investigación es útil porque ha brindado insumos a la URACCAN, para realizar la readecuación curricular de la carrera de Informática Administrativa.

Palabras clave: Pertinencia del currículo, carrera Informática administrativa, dimensión externa e interna.

\section{Summary}

The pertinence of the curriculum of the Administrative Computing career at the University of the Autonomous Regions of the Caribbean Coast of Nicaragua (URACCAN), Bilwi Campus, has been investigated. The study was qualitative, evaluative and transversal; the purpose was to study the pertinence of the curriculum of this career in the campus. The data collection techniques were based on interviews, focus groups and document review. For information processing matrix table was used to make a critical analysis of the results.

\footnotetext{
[1] Coordinadora de Carreras de Administración e Informática Recinto Bilwi. Máster en Docencia Universitaria. Autora. ivonne_mclean2003@yahoo.com

[2] Docente en Biología Recinto Bilwi. Máster en Salud Pública. shooker55@yahoo.com
} 
The main finding of the study has shown that the curriculum of the career of Administrative Computing has several positive aspects. However, this is irrelevant, since the evaluation of the external dimension is pertinent, contrary to the internal and administrative dimensions. As the three dimensions should be closely interrelated and in the absence of one of these dimensions it affects the pertinence of the entire curriculum. This research is useful because it has provided inputs to URACCAN, in order to do the curricular readjustment of the Administrative Computing career.

Keywords: Curricular pertinence, administrative computing career, internal and external dimension.

\section{Introducción}

Los cambios globales, nacionales y locales que se desarrollan en las últimas décadas, afectan de manera particular el ámbito educativo y a lo cual no está exenta la URACCAN, como una Universidad de Educación Superior y en particular la carrera de Informática Administrativa, ya que la educación es factor clave y decisivo para el desarrollo humano y su incidencia en la vida política, económica y social.

La URACCAN rige su funcionamiento bajo una misión, visión, principios y valores que abarcan todo el accionar institucional, como una universidad comunitaria intercultural al servicio de los pueblos indígenas y afrodescendientes, que han reivindicado sus derechos en el proceso de construcción de las Regiones Autónomas multiétnicas, en el marco del reconocimiento y ejercicio de los derechos humanos y colectivos.

La URACCAN recinto Bilwi, en cumplimiento de su misión y visión, apertura la carrera de Técnico Superior en Informática Administrativa en el año 2000, a fin de dar respuesta a las demandas de la población en un mundo globalizado que requiere la información de manera automatizada, agilizando procesos de control, almacenamiento y obtención de información con mayor rapidez. Es una de las carreras afines a la tecnología con mayor demanda a nivel nacional, regional y local.

Debido a esta demanda, se dio paso a una primera revisión del currículo del Técnico Superior en el año 2004 y se logró diseñar la licenciatura en Informática Administrativa, dando la oportunidad a que los egresados del Técnico Superior pudiesen insertarse a este nuevo plan de estudio. No obstante, en el recinto Bilwi en el año 2007, se realizó una adecuación al plan de estudio dando como resultado salida a dos niveles de egreso: Técnico Superior y la licenciatura en Informática Administrativa, es así que el currículo está vigente desde el 2004, sin ninguna readecuación o actualización curricular.

En las evaluaciones constantes a los programas de asignaturas desde el 2004, en el proceso de seguimiento a la carrera por parte del Consejo Nacional de Universidades (CNU), en el año 2007 en el Recinto Nueva Guinea, éstas reflejan ciertas debilidades, 
principalmente en cuanto a la desactualización del currículo; no obstante, que la informática es una ciencia que avanza rápidamente. Desde el año 2007 se trabaja en una propuesta de adecuación al currículo que aún no se concreta.

El propósito principal de esta investigación es determinar la pertinencia del currículo en la carrera de licenciatura en Informática Administrativa ofertada en el recinto de Bilwi, a fin de evaluar los elementos estratégicos para fortalecer la revisión y readecuación curricular.

\section{Metodología}

Es un estudio de tipo cualitativo, evaluativo, ya que se buscaron opiniones para comprender la oferta, demanda y calidad educativa en la carrera de Informática Administrativa, asociado a la valoración de la pertinencia del currículo de la carrera 2004 - 2009, desarrollado en la URACCAN Recinto Bilwi, es de corte transvesal, realizada entre julio 2009 - agosto 2010.

El universo muestral estuvo compuesto por: 5 empleadores, cien por ciento de los posibles empleadores; 8 graduados (4 de cada generación), 6 docentes (3 informáticos y 3 administrativos), 8 estudiantes de la carrera de Informática Administrativa (4 mujeres y 4 varones, 2 de cada sección), secretaria académica del Recinto Bilwi, bibliotecaria, administradora, responsable de Recursos Humanos.

Esta muestra fue seleccionada por conveniencia, debido a las razones siguientes:

- Dispersión de la población.

- Costos en la aplicación de los instrumentos para la recopilación de la información.

Las técnicas de recolección de información fueron entrevistas, grupo focal y revisión documental. De igual manera, la fuente de recolección de información primaria fue a todo este grupo de personas mencionados anteriormente, así como también, la revisión del currículo de la carrera de Informática Administrativa, evaluación a los programas de asignaturas y como fuente secundaria fueron: bibliografías y documentos relacionados al tema de investigación.

Para la tabulación y procesamiento de la información, se utilizaron tablas matriciales para el procesamiento, ordenamiento y clasificación de acuerdo a preguntas realizadas y frecuencia de respuestas obtenidas.

En relación al criterio ético, quienes brindaron información para la realización de este trabajo sus nombres no se mencionaron en ninguna de las fases de la investigación, salvo por autorización previa. 


\section{Resultados y discusión}

En este proceso investigativo se evaluó la pertinencia del currículo de la carrera de Informática Administrativa, URACCAN Recinto Bilwi, tomando en cuenta las tres dimensiones: interna, externa y administrativa.

En esta síntesis se retoman los aspectos más relevantes de esta investigación. En este sentido en el currículo se encontró lo siguiente:

- Vigente desde el 2004.

- Modalidad presencial.

- Régimen cuatrimestral.

- Dos formas de egreso.

- Duración 3 años y medio.

- Plan de estudio:

- Centrado en asignaturas.

- Distribuido en áreas: $6 \%$ básico, 31\% profesional y 63\% especialización.

- Requisitos de admisión.

- Modalidad de egreso.

- Personal docente.

- Laboratorios (2 de informática y 1 de arquitectura) con equipos que tienen las características tarjeta madre ASROCK, memorias DDR2 de un giga, disco duro de 80 gigas, microprocesador Intel Pentium dual Core Inside.

Al describir el currículo se consideró oportuno valorarlo según lo normado por la Comisión Académica del Consejo Nacional de Universidades (CNU), el cual está agrupado en tres aspectos que se corresponden con el currículo en estudio. Sin embargo, el currículo requiere ampliar algunos aspectos y ser más específicos, en cuanto a: justificación, fundamentación desde una visión interna, externa y administrativa que responda a la pertinencia y demanda de la carrera.

A pesar de contar con parte de los requerimientos y en algunos casos escasos, no es suficiente.

En cuanto a la valoración de la pertinencia interna, externa y administrativa:

En la dimensión interna es evidente la interrelación y coherencia que existe entre la misión y visión de la URACCAN con los objetivos del currículo de la carrera de Informática Administrativa, ya que la misión es: "formar recursos humanos con conocimientos, capacidades científico-técnicas, actitudes humanistas, sentido del emprendimiento y la innovación, que contribuyen al fortalecimiento del sistema autónomico regional y del país." y en los objetivos de la carrera plantea: "formación 
de profesionales en el campo de la computación con una alta calificación y un gran sentido de responsabilidad social, técnicamente capaces de enfrentar los retos y avances de la ciencia de la computación y la administración, con una visión integral en los campos académicos, social y humanístico".

Al hablar de responsabilidad social del pensum de una carrera que se está evaluando su pertinencia, podemos citar a Tünnerman C. (2009) quien precisa:

La pertinencia de la Educación Superior ha evolucionado hacia una concepción amplia de la misma y a su estrecha vinculación con la calidad, la equidad, la responsabilidad social. Todo esto apunta a fortalecer la convicción de que las instituciones de educación superior tienen una ineludible responsabilidad social y no sólo académica y profesional. Y, lo más importante, es que dicha responsabilidad social, en última instancia, es la que realmente determina su pertinencia y calidad.

Si se continua con el análisis del currículo del 2004, en la carrera de Informática Administrativa se observa que en la justificación planteada expresa que:

Ante el avance tecnológico, se hace necesario la formación, capacitación y actualización de los recursos humanos en la especialidad de licenciatura en Informática Administrativa, que sean capaces de enfrentar los avances tecnológicos con una alta calificación y un gran sentido de responsabilidad social...

Pero, en el mismo currículo, ni la justificación, ni los objetivos de la carrera se visualizan en los contenidos de algunos programas de la especialidad, tales como: Introducción a la programación, programación I, programación gráfica, programación orientada a objetos, base de datos, análisis y diseño de sistemas, mantenimiento y reparación de PC, entre otros, ya que frente a las exigencias globales, y especialmente ante esta ciencia tecnológica que avanza tan vertiginosa, se enfrenta a unos programas congelados en el tiempo, lo que hace que ofertemos un currículo desactualizado de hace seis años, y la realidad del avance de la informática es otra.

Esto también se refuerza si tomamos en cuenta a Quesada et al (2001), al referirse a la relación que debe existir con los fines, la misión de la universidad y las instancias que proponen una carrera: facultad, centro o sede y unidad académica.

En este sentido, se afirma que es pertinente la justificación y los objetivos del currículo con la visión y misión de la universidad. Sin embargo, los programas que son el verdadero nexo con los estudiantes para su formación académica y profesional no responden al avance tecnológico y a las necesidades del entorno. 
Sin embargo, los currículos de educación intercultural para ser pertinente deben estar basados en relaciones horizontales donde prevalece el diálogo, a través del cual se propicia el conocimiento mutuo, la comprensión, el respeto, el intercambio y la solidaridad entre los pueblos y las culturas.

Aguado, expresa que:

una educación intercultural es un enfoque educativo basado en el respeto y valoración de la diversidad cultural, dirigido a todos y cada uno de los miembros de la sociedad en su conjunto, que propone un modelo de intervención, formal e informal, holístico, integrado, configurador de todas las dimensiones del proceso educativo en orden a lograr la igualdad de oportunidades/resultados, la superación del racismo en sus diversas manifestaciones, la comunicación y competencia intercultural.

La educación intercultural pretende formar en todos los estudiantes una competencia cultural madura; es decir, un bagaje de actitudes que les capacite para funcionar adecuadamente en nuestra sociedad multiétnica, multicultural y multilingüe.

Sáez (2001) explica que un currículo intercultural es un currículo abierto a las experiencias, diferencias, intereses y expectativas de aquellos que lo viven cada día. Un currículo que garantiza la calidad de conocimientos para todos.

La finalidad es desarrollar un currículo integral, donde el ser humano tenga una formación digna, solidaria, con autodeterminación, con excelencia académica en busca de transformar la sociedad en un lugar donde todo ser humano pueda vivir dignamente (Villarini, 2002).

Es así, que el currículo de la carrera de Informática Administrativa sólo menciona en sus objetivos educativos el aspecto de la interculturalidad como eje transversal de la universidad; sin embargo, no orienta en su metodología como realizar acciones para el logro de estos objetivos educativos. La metodología de todo el proceso educativo y su sistema de evaluación, no brinda orientaciones específicas que permita y apoye al docente para el desarrollo de una educación intercultural que atienda las diferencias, intereses, expectativas y desarrolle una competencia cultural madura en los futuros profesionales de esta ciencia. Por tanto, mientras más explícito aborde estos aspectos el currículo, se podría hablar de un currículo integral.

El contexto muestra que el currículo está desactualizado y cuando los graduados salen al mercado laboral se están enfrentando a esta desactualización, por lo que es necesario que el currículo prepare estudiantes para la vida, o sea que con su formación académica y profesional ellos tengan capacidad de innovar y ser emprendedores, lo cual debería ser uno de los ejes transversales de todo el currículo. 
Los resultados de la evaluación de la carrera mediante entrevista y grupo focal, en su mayoría expresaron algunas fortalezas y debilidades en cuanto a: cuerpo docente y currículo, resumiéndolo en la tabla siguiente:

\begin{tabular}{|c|c|}
\hline Fortalezas & Debilidadades \\
\hline $\begin{array}{l}\text { Docencia } \\
\text { - Jóvenes, dispuesto al trabajo, desde } \\
\text { la práctica se vienen apropiado con la } \\
\text { misión y visión de la universidad. } \\
\text { - Excelentes docentes. }\end{array}$ & $\begin{array}{l}\text { Docencia } \\
\text { - No tienen especialización. } \\
\text { - Requieren formación psicopedagógica y } \\
\text { poca experiencia en investigación }\end{array}$ \\
\hline $\begin{array}{l}\text { Currículo } \\
\text { - Enfocado en dos áreas. } \\
\text { - Duración y modalidad. } \\
\text { - Dos niveles de egreso. }\end{array}$ & \begin{tabular}{l}
\multicolumn{1}{c}{ Currículo } \\
- Desactualizado. \\
- Metodologías más integrales. \\
- Poca práctica.
\end{tabular} \\
\hline
\end{tabular}

En entrevista a la secretaria académica opina que los docentes:

son recursos humanos jóvenes, desarrollando diferentes tareas, hay disponibilidad de personal dispuesto al trabajo que desde la práctica se viene apropiando de la misión y visión de URACCAN, del modelo pedagógico, con compromisos hacia el proceso autonómico y varios miembros del personal docente de la carrera están por concluir una maestría.

Sin embargo, la gran mayoría del personal docente no tiene especialización en el campo de la informática, requiere de formación metodológica, poca experiencia en la investigación, la publicación requiere de mayores esfuerzos y gestión, puesto que hay material elaborado, pero no se publica ni se divulga. Se han hecho investigaciones, pero se requiere una revisión profunda de las metodologías de investigación implementadas.

A la vez los graduados expresan: Es importante subrayar que la carrera cuenta con excelentes docentes, pero a la vez hay que enmarcar que existen algunos docentes a quienes se les asigna la tarea de impartir algunas asignaturas y estos no tienen la capacidad para llevar a cabo dicha tarea, cabe mencionar que esto puede ser por falta de conocimiento o por metodología.

Los docentes y estudiantes coinciden en que:

"Hay insuficiente personal docente de tiempo completo y es notoria la sobrecarga de funciones en estos docentes y en algunos casos se contratan docentes sin experiencia y no cuentan con ningún método pedagógico" por otro lado, expresaban que: "hay muy pocos docentes especializados y con experiencia en el ramo, hay poco estimulo y motivación para docentes y estudiantes en cuanto a los trabajos que realizan". 
Si se coteja estos resultados con lo planteado por Carlos Tünnermann. en el documento "El rol del docente en la educación superior del siglo XXI", se visualiza que el rol del docente, ya no es el del catedrático que simplemente dicta o imparte clases magistrales y luego califica los exámenes de sus estudiantes, hoy día, el profesor universitario es fundamentalmente un diseñador de métodos de aprendizaje, un suscitador de situaciones o ambientes de aprendizaje y que participa con sus estudiantes en la maravillosa aventura del espíritu que es descubrir y difundir el conocimiento, mediante la investigación.

El análisis en cuanto a los mecanismos y sistemas de integración, se concreta en que no existen planes, ni programas que integren la docencia, investigación y extensión. A pesar que hay esfuerzos por parte de la universidad, aún hace falta capacitar, especializar a los docentes, promover la investigación de forma sistematizada y planificada.

En cuanto a publicación y extensión que se realiza desde la carrera, la secretaria académica expresaba: "Los docentes y estudiantes más que todo se integran a la actividad de investigación a través de las tutorías o elaboración de monografías o de trabajos para presentar en "La Ocasión".

Continúa explicando:

El trabajo de extensión si bien no se planifica como tal, sí se logra articular a través de las prácticas y trabajos de terreno. Me parece que debiera realizarse una valoración de la extensión como tal y las alternativas con las cuales se pudiera realizar. Hay planes de trabajo. Lo que se necesita es revisarlos y darles un enfoque integral. Para elaborar planes más objetivos se requiere del establecimiento de convenios interinstitucionales que ayuden a una mejor práctica de los estudiantes.

El planteamiento anterior por la secretaria académica coincide con el informe del Consejo Nacional de Universidades (CNU), el cual plantea:

La carrera carece de una sólida y sistemática organización que promueva el fomento de la investigación tanto de sus docentes como de sus estudiantes. La expresión estudiantil en investigación son los proyectos en la JUDC en la que sus contenidos están ligados a resolver situaciones particulares de la empresa local, la masificación de estas iniciativas es aún insuficiente.

Los estudiantes realizan en diferentes asignaturas trabajos de fin de curso, trabajos de terreno y los mejores calificados son seleccionados para compartirlos en la "Ocasión" jornada para construir y compartir conocimientos, en el que presentan 
sus diferentes proyectos, tales como sistemas, sitios web, los cuales son enviados a biblioteca y participan en la Jornada Científica a nivel nacional.

Siguiendo con la evaluación de la carrera han relucido algunas fortalezas y debilidades en cuanto a recursos materiales, bibliográficos y tecnológicos, en el que docentes y estudiantes entrevistados coinciden en:

Contamos con laboratorio de computación amplio, herramientas y capacidad para realizar prácticas, tenemos aula, biblioteca, bibliografía; no obstante, es limitada en muchos casos un solo ejemplar, se cuenta con un cyber para realizar nuestros trabajos investigativos; sin embargo, faltan equipos en relación a la cantidad de estudiantes, se cuenta con máquinas en mal estado, no se cuenta con todos los medios y recursos tecnológicos para el cumplimiento de algunas clases prácticas, se tiene acceso a internet, pero pocos equipos están conectados a la red. Continúan expresando que no cuentan con la: "disponibilidad de software: el docente debe buscarlos, existen pocos o ningún programa informático para las demás asignaturas como contabilidad, administración y otros que sirvan para la práctica y se trabaja con software sin licencia (piratería).

Constituyen estos recursos, elementos primordiales para la ejecución de un currículo competitivo, es imprescindible que docentes y estudiantes dispongan de los mismos, porque permite desarrollar procesos educativos en ambientes efectivos, productivos, accesible, actualizados y es así y sólo así que los estudiantes pueden desarrollar plenamente sus capacidades para el desempeño profesional, siendo facilitado el proceso por su docente.

Aún es un tanto más preocupante cuando refieren que la carrera no cuenta con software, los docentes deben rebuscarlos, siendo estos ilegales o pirateados o bien software de prueba "DEMO"; como parte del componente tecnológico, el software es una herramienta fundamental y básica para el desarrollo de una carrera tecnológica. En el estudio de factibilidad de la carrera se debió haber asegurado estos aspectos antes de ofertar la carrera, si queremos asegurar la excelencia académica.

Al no contar con estos recursos tecnológicos, tanto hardware como software, implica realizar trabajos inseguros, no confiables, ineficaz e ineficiente, las razones son muchas, según Abadía (2006), el trabajar con software no legalizado conlleva a varias desventajas, los cuales se vuelven en ventajas si es legalizado "acceso a soporte técnico, derecho a la actualización de las últimas versiones y paquetes de mantenimiento (parches), descuentos en la renovación de licenciamiento y seguro del buen funcionamiento" y por supuesto las garantías. 
Al revisar el informe de seguimiento que hiciere el CNU al currículo de la carrera de informática administrativa en Nueva Guinea en el año 2007, este expresa algunos aspectos, tales como:

La carrera no cuenta con el suficiente aseguramiento tecnológico; aún hacen falta adquirir como mínimo, un laboratorio adicional de informática, un servidor, una red, el uso generalizado de Internet por toda la comunidad, que permita fácil acceso a las TIC para que se potencie el aprendizaje. El diseño curricular presenta algunas limitaciones, por lo que es necesario su revisión y reformulación, tanto técnica como metodológica. No puede apreciarse el balance entre teoría y práctica en cada asignatura, careciendo de orientaciones metodológicas en la evaluación que se requiere para cada asignatura, por lo que es uniforme e incompleta en todos los programas.

El recinto de Nueva Guinea tiene otras particularidades, este informe ha sido muy valioso para la carrera, ya que es el mismo currículo ofertándose en el recinto Bilwi. Este enunciado está muy relacionado con la realidad y las condiciones que enfrenta la carrera en Bilwi, ya que de igual forma se dispone de muy pocos recursos materiales, bibliográficos y tecnológicos en relación a la cantidad de estudiantes.

La carrera cuenta con aproximadamente ciento veinte estudiantes, quienes son de diversas etnias: miskitus mayoritariamente, luego hay una representación de mestizos, creoles y minoritariamente los sumo mayangna. Más del $60 \%$ son mujeres y el otro porcentaje son varones.

Los laboratorios de informática cuenta con aproximadamente 25 computadoras y en cambio se atiende hasta 45 estudiantes por grupo, y ante esto los laboratorios no dan abasto, situación que el docente debe enfrentar y es difícil brindar atención individualizada y personalizada. Con esta cantidad de estudiantes y computadoras (dos y hasta tres estudiantes deben compartir una computadora), cuando éstas son herramientas básicas para esta carrera tecnológica.

Cabe mencionar que estos laboratorios son utilizados por todos los estudiantes de todas las carreras ofertadas en el Recinto Bilwi, esto significa que la demanda en la utilización de los laboratorios es aún mayor. Ante esto el REDICA/CSUCA/BID (2002) refiere que los "Laboratorios para el Área Informática debe contar con suficientes terminales de cómputo para que no más de 2 estudiantes compartan cada una en clase”. Esto implica que si la carrera es evaluada en este momento para su acreditación, sería esto un aspecto negativo, uno de los elementos principales que está repercutiendo en el rendimiento académico de los estudiantes de esta carrera.

Otro componente importante en la dimensión interna es la relación teoría práctica del plan de estudio del currículo, en el que todos los docentes entrevistados 
expresaron que las asignaturas que imparten "son pertinentes o son de mucha importancia y necesarias, están acorde al perfil de la carrera y tipo de profesional que quiere según ejes transversales, objetivos de la universidad, y sí existe relación entre la teoría y la práctica". No obstante, consideran algunas debilidades en ciertos programas de asignaturas, tales como:

Requiere revisión en cuanto al orden lógico entre uno y otro programa de asignatura, hay redundancia en contenidos, ejemplo programación I y II, sobrecargado de contenidos, auditoría en informática, requiere revisión en la recomendación metodológica de Administración de Centros de Cómputo, debería recomendar trabajo de fin de curso, la mayoría hay que actualizarlos, implementar el uso de programas informáticos aplicados a la contabilidad.

Para continuar con el análisis del currículo de la carrera de Informática Administrativa tomamos en cuenta lo que dice Quesada (2001), sobre la pertinencia externa cuando refiere que atiende los aspectos relacionados con el contexto social y profesional de la carrera, tratándose de las necesidades sociales y laborales que justifican la carrera.

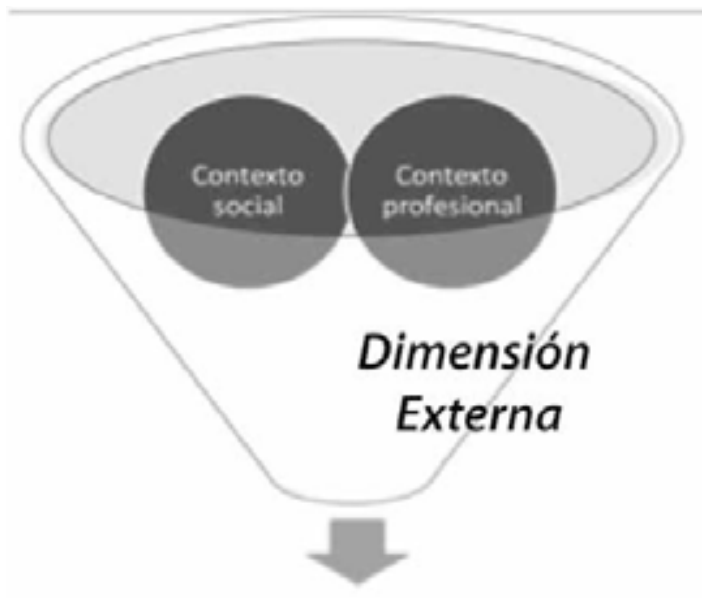

Las entrevistas realizadas a posibles empleadores, brindaron las siguientes informaciones en cuanto a: el contexto profesional en el que se encuentran algunas necesidades laborales en el campo de las Tecnologías de Información y Comunicación y se encontró aproximadamente 90 institucionales entre privadas, públicas y educativas en Bilwi, que requieren de un promedio de dos informáticos por institución, la mayoría de ellos de forma permanente y en una menor cantidad de forma temporal. 
En relación a esta necesidad laboral en Bilwi, consideran que es pertinente la oferta de esta carrera, ya que los posibles empleadores opinan que los cargos que pudieran ocupar los profesionales en Informática Administrativa son amplios, ya que lo visualizan como dos ciencias por separado, la mayoría de las instituciones requieren Informáticos puros y una minoría a Informáticos con conocimientos administrativos. Siendo estos cargos en la parte administrativa, los siguientes: asistente administrativo, administrador, Caja, servicio al cliente, asistente del gerente, Contador auxiliar, secretaria /o y responsable de Recursos Humanos.

Al hacer el análisis de lo expresado por los posibles empleadores llama la atención que a pesar de que la mayoría refieren que requieren de informáticos puros, hacen énfasis en los cargos administrativos y áreas especificas donde podrían laborar, lo que se podría interpretar como uno de los aspectos positivos de este currículo, al preparar licenciados (as) en Informática Administrativa o sea Informáticos con ciertos conocimientos administrativos que le facilita su inserción en el campo laboral o el establecimiento de su propia microempresa informática.

Al continuar las entrevistas con los posibles empleadores, refieren que lo que ellos esperan de un informático es que ocupen cargos de: soporte técnico, director de Área de Informática, director de los sistemas de información y base de datos, administrador de redes, responsable de estadística, diseñador y programador Web, docente de informática, programador y luego hicieron la lista de las funciones de cada uno de los cargos.

Se considera que las funciones que plantean los posibles empleadores se podrían retomar al hacer la revisión curricular, ya que en una revisión para adecuación hay que recordar que lo que se hace es una actualización, inclusión o eliminación de contenidos con que cuenta el currículo y esto podría ser la oportunidad de adecuar el currículo para responder a las instituciones y a la sociedad en general, así también responder al objetivo del currículo de esta carrera que es la:

Formación de profesionales en el campo de la computación con una alta calificación y un gran sentido de responsabilidad social, técnicamente capaces de enfrentar los retos y avances de la ciencia de la computación y la administración, que les permita desarrollar procesos tecnológicos y administrativos, con una visión integral en los campos académicos, social y humanístico.

Habría que hacer un balance de las demandas actuales y futuras que tienen los empleadores y posibles empleadores de la región con las necesidades nacionales y globales como referentes para la actualización del currículo de Informática Administrativa.

Si seguimos este análisis con los resultados de las entrevistas realizados a graduados que están laborando, se logra conocer que la mayoría, el 69\% están laborando y 31\% 
que no trabaja actualmente. Asimismo, aprovechamos en conocer en qué área se están desempeñando y la mayoría expresaron estar laborando en la parte administrativa, una minoría en docencia y en la parte de informática pura.

En cuanto a lo que manifestaron en entrevista los empleadores, solamente un $40 \%$ de los graduados está laborando en el área administrativa y la mayoría de los egresados trabajan en el área de informática, estando en contradicción con lo que manifiestan los graduados entrevistados, quienes dijeron que la mayoría está en el área administrativa. Todo esto, nos hace reflexionar en que los empleadores están aprovechando de los conocimientos administrativos e informáticos de los graduados, ya que aunque están en áreas administrativas, están utilizando diferentes tipos de programas informáticos para realizar las funciones encomendadas, nuevamente resaltan las bondades de este currículo para la inserción en el mundo laboral.

Frente a las fortalezas de la carrera han expresado los graduados:

Amplios conocimientos en manejo de las computadoras y conocimientos básicos de sistemas automatizados, contabilidad y administración, cuentan con dominio en computación y capacidad de análisis, con conocimientos teóricos.

Hay que tomar en cuenta y contrastar con las limitaciones que también expresaron, como son: "poca práctica en las materias, las pocas horas prácticas inciden en que el profesional no se desempeñe con eficiencia en las funciones, debido al conocimiento práctico limitado.

Y los empleadores expresaron:

No ejerce su función como informático, sino como administrativo, algunas actividades del sistema no las maneja, pero las va superando a medida que lo pone en práctica, pocas habilidades en cuanto a la reparación y mantenimiento de PC, y debilidades en conocimientos administrativos.

Si junto a esto asociamos la limitante planteada por los empleadores como la: "necesidad de actualización de conocimientos tecnológicos, conocimientos de sistemas computacionales, estadística y administración", podríamos tener una referencia para lograr la pertinencia externa relacionada con el contexto social y profesional de la carrera, tratándose de las necesidades sociales y laborales que justifican la carrera de Informática Administrativa. 
En cuanto a la evaluación externa del currículo y la oferta con las carreras similares al área de Informática en todo el país, se afronta una inclinación por la Ingeniería de la Computación y Sistemas de Información, por ejemplo Ingeniería en Sistemas de Información, Licenciatura en Informática Educativa, Ingeniería en Administración de Sistemas Informáticos, entre otras y es la URACCAN la única universidad formadora de recursos humanos en la especialidad de "Licenciatura en Informática Administrativa".

La única competencia directa en la región, es Ingeniería en Sistemas como oferta afín, pero recalcando que ambas son carreras emergentes, tienen proyección hacia el futuro y cuyos espacios ocupacionales se refieren a las necesidades de la sociedad en general y en especial para el área de las TIC, que permiten a las organizaciones contar con profesionales que tengan la posibilidad de ser productivos utilizando la tecnología informática de la mejor manera.

Según Mora, S. (2004), quien retoma a Mata (2003), plantea que:

de acuerdo con los estudios nacionales e internacionales, así como la experiencia y criterios de profesionales y docentes, en que se basa el desarrollo de un rediseño curricular, los espacios ocupacionales emergentes son: Ingenieros en sistemas de información, programadores, especialistas en telecomunicaciones, administradores de bases de datos o de información, Administradores de proyectos y desarrolladores de aplicaciones y de estos estudios se deriva una clara tendencia a que los centros de enseñanza especialicen más al profesional graduado, que ya no se formen con una visión global de "todo", lo cual es claro que en esta área del saber, cada vez es más difícil, sino que se busque la formación más especializada de los profesionales, con una mayor profundidad, obviamente en el área de la especialidad.

Si continuamos con la evaluación de la dimensión administrativa tomando en cuenta la disponibilidad de recursos en la carrera de Informática, la entrevista con la administradora de la URACCAN da como resultado de que si existe un presupuesto anual asignado a la universidad por el Ministerio de Hacienda y Crédito Público, que es ejecutado únicamente para pago de salarios (docencia), por lo que es preciso utilizar fondos propios de la universidad, para cubrir los otros gastos como son compra de materiales didácticos, pago de tutoría, jurado, trabajos de terreno, entre otros.

Ante esto, la carrera desarrolla actividades para asegurar su funcionamiento, es así que oferta cursos alternos, como cursos libres de operador y de mantenimiento y reparación de microcomputadoras a la población en general, los cuales aportan fondos propios a la carrera y trata de asegurar ciertas necesidades básicas para el proceso educativo, como por ejemplo: compra de piezas de computadoras y mantener los equipos de cómputo en buen estado, el cual contribuye a realizar las prácticas de los estudiantes. 
Tabla que resume la disponibilidad de los recursos:

\begin{tabular}{|c|l|}
\hline \multicolumn{1}{|c|}{ Recursos } & \multicolumn{1}{c|}{ Disponibilidad } \\
\hline Económico & $\begin{array}{l}\text { Existe un presupuesto asignado a la carrera, básicamente sólo } \\
\text { cubre el pago de docentes. } \\
\text { Con fondos propios se cubre otros gastos como: tutoría, mate- } \\
\text { riales didácticos, entre otros. La carrera oferta cursos alternos, } \\
\text { los cuales generasn fondos propios. }\end{array}$ \\
\hline Bibliográfico & $\begin{array}{l}\text { Se dispone de la bibliografía básica de forma impresa, aunque } \\
\text { es insuficiente, relativamente muy poco a la cantidad de estu- } \\
\text { diantes (de 400, 114 son actualizados). } \\
\text { REDICA/CSUCA (4 títulos-6 ejemplares para cada curso). }\end{array}$ \\
\hline Infraestructura física y tecnológica & $\begin{array}{l}\text { No se cuenta con bibliografía en forma digital. } \\
\text { En infraestructura existe lo básico, falta de pupitres, no se } \\
\text { cuenta con SW legalizado, se cuenta con } 3 \text { laboratorios (2 } \\
\text { informática y 1 de arquitectura). }\end{array}$ \\
\hline
\end{tabular}

Docentes y estudiantes entrevistados y en grupo focal coinciden en que:

Existe el acceso a diversos tipos de bibliografías, pero algunas están desactualizadas e insuficiente; sin embargo, se tiene la posibilidad de acceder a servicio de Internet para obtener información que se necesite con respecto a los temas de investigación y esto facilita el acceso para buscar libros en Internet.

Con respecto a la estructura física y tecnológica con la que cuenta la carrera expresaba la secretaria académica:

Hay lo básico para el desarrollo de la carrera; empero, hay que considerar que en ese campo de la tecnología hay cambios, transformaciones muy rápidas por lo que se requieren de muchísimos más recursos para mantener actualizado el equipamiento de la carrera.

El cuanto a software, los docentes entrevistados expresaron:

Se cuenta con los programas básicos, en su mayoría son de la Microsoft y actualmente se realizan esfuerzos de implementar programas de código abierto, y que hasta ahora se empieza a utilizar, tenemos una asignatura de sistemas operativos en la cual se ve Linux y a partir de allí se está promoviendo el uso de software libre.

En grupo focal con los docentes refieren que la Universidad no proporciona software; no obstante, los docentes obtienen estos (pirateados, bajados de internet, demo), los cuales se aplican en el proceso educativo, ya que la universidad no tiene ningún programa con licencia. 
Los recursos humanos académicos con que cuenta la carrera, en su minoría con docentes de tiempo completo, según la responsable de Recursos Humanos y su perfil profesional es: Ingeniería en Sistemas con especialización en TIC, licenciatura en Informática Administrativa, licenciatura en Administración de Empresas con maestría en Docencia Universitaria, licenciatura en Administración de Empresas con maestría en Administración Pública y licenciatura en Informática finalizando su maestría en Docencia Universitaria.

Como se puede apreciar aquí, la carrera no cuenta con un plan de desarrollo y promoción de los recursos académicos, ni mecanismos que faciliten la actualización sistemática de los académicos y la renovación de sus conocimientos informáticos y al ser graduados de la misma universidad, muchos de los docentes tienden a reproducir sus esquemas y metodologías de aprendizaje, esta carrera no tiene una organización dirigida a la investigación basada en la planificación sistemática y el estímulo que lo fomente, tanto en docentes como en estudiantes.

Medidas administrativas del currículo: se evalúa constantemente los programas de asignaturas; sin embargo, aún no ha servido para la actualización del mismo. En cuanto a las estrategias de coordinación: interuniversitaria, intrauniversitaria, sector público y privado, existen pocas coordinaciones que permitan realizar pasantías o promover aun más la función de extensión.

Con todo el análisis, el hallazgo principal del estudio refleja que el currículo de la carrera de Informática Administrativa, tiene varios aspectos positivos; antes bien hay retos que alcanzar. En la evaluación de la dimensión interna y administrativa se reflejó que no es pertinente, a pesar de que la dimensión externa sí. Como las tres dimensiones deben estar íntimamente interrelacionados y al no tener congruencia una de estas dimensiones afecta la pertinencia de todo el currículo de la carrera.

\section{Conclusiones}

\section{En cuanto a la pertinencia interna:}

1. Es evidente la interrelación y coherencia que existe entre la misión y visión de la URACCAN con los objetivos del currículo de la carrera de Informática Administrativa, pero el contenido de algunas asignaturas del Plan de Estudio del currículo ha quedado congelado en el tiempo.

2. A pesar de enfrentarnos ante un currículo desactualizado se resaltan algunos aspectos positivos, está enfocado en dos áreas: la informática y la administrativa, duración de tres años y medio por su modalidad cuatrimestral y le da salida a dos niveles de egreso (Técnico Superior y Licenciatura). 
3. No se ha potencializado el sentido del emprendimiento e innovación, un sistema de evaluación más integral, metodologías como es el enfoque de Aprendizaje Basado en Problemas ABP, y otras metodología participativas, como ejes transversales del currículo.

4. En el currículo apenas se visualiza el aspecto de la interculturalidad en los objetivos específicos; sin embargo, en la metodología no especifica cómo lograr estos objetivos educativos.

5. En el currículo, los descriptores no especifican la cantidad de horas prácticas por asignatura, lo único que detalla son las horas de trabajo de terreno en cuatro asignaturas de la especialidad.

6. La mayoría de los docentes de la especialidad, tienen poca experiencia laboral, no tienen ningún curso de especialización, tampoco tienen formación pedagógica o metodológica y poca experiencia en la investigación.

7. Existen muy pocos trabajos investigativo de docentes y estudiantes de la carrera de Informática Administrativa, no existe un plan de investigación y aunque existe una política institucional de investigación no se ve concretizado.

\section{En cuanto a la pertinencia externa:}

8. Existen aproximadamente noventa instituciones gubernamentales y no gubernamentales que requieren de los servicios de profesionales informáticos, en un promedio de dos informáticos por institución.

9. Actualmente los empleadores están aprovechando de los conocimientos administrativos e Informáticos de los graduados, ya que aunque están en áreas administrativas, están utilizando diferentes tipos de software para realizar las funciones encomendadas.

10. La URACCAN es la única universidad del Caribe que oferta la carrera de "Licenciatura en Informática Administrativa", siendo una carrera emergente y cuyos espacios ocupacionales son las necesidades de la sociedad.

11. Todo lo relacionado a la pertinencia externa refleja que el currículo de la carrera de Informática Administrativa es pertinente, ya que existe demanda de estos profesionales en la región.

\section{En cuanto a la pertinencia Administrativa:}

12. El 6\% otorgado por el Consejo Nacional de Universidades (CNU), sólo cubre el pago de los docentes. URACCAN de sus fondos propio cubre otros requerimientos básicos (pago tutoría, compra de materiales didácticos, entre otros) y este recurso económico está sujeto a recortes y reajustes.

13. Existe una estructura física básica, con poca iluminación, falta de pupitres, poca ventilación, con capacidad para pocos estudiantes, por lo cual, la estructura docente no cumple con una adecuada higiene escolar. 
14. En relación a recurso bibliográfico impreso es insuficiente. aunque se cuenta con la conexión a Internet.

15. En relación al recurso tecnológico no se cuenta con software, hay pocos equipos en relación al número de estudiantes (aproximadamente 3 estudiantes por máquina) y son insuficiente e incompletas las herramientas existentes.

16. La carrera no cuenta con un plan de desarrollo y promoción, ni mecanismos de renovación de conocimientos informáticos que faciliten la actualización sistemática de los recursos académicos. Tampoco existe ningún curso, especialización o postgrado diseñado para el graduado de esta carrera.

17. No existen convenios, ni acuerdos firmados con otras universidades, instituciones estatales, privadas y con unidades académicas similares de la carrera.

18. En el análisis de la evaluación del currículo de la carrera de Informática Administrativa, se determinó que no es pertinente, ya que a pesar que la dimensión externa resultó pertinente, en su dimensión interna y administrativa no fue así. Estas dimensiones están íntimamente interrelacionadas $y$ al no tener pertinencia una de estas dimensiones afecta la pertinencia de todo el currículo de la carrera.

\section{Recomendaciones}

1. Que la URACCAN del Recinto Bilwi a través de su secretaria académica oriente la formación de un equipo para la revisión del currículo para su adecuación hacia un currículo pertinente.

2. Que este equipo formado, tome en cuenta los aspectos positivos que tiene actualmente el currículo, incluir las líneas de investigación, las consideraciones del sector social y profesional, que en los descriptores se detallen la cantidad de horas teóricas-prácticas y establecer la evaluación del currículo de forma sistemática, ya que es una carrera emergente y la tecnología de la información avanza con rapidez.

3. Al hacer la adecuación curricular que el equipo valore la importancia de: el enfoque intercultural, el emprendimiento e innovación como ejes transversales, que incluyan metodologías más integrales, como por ejemplo: metodologías de Aprendizaje Basado en Problemas (ABP) y otras metodologías participativas que orienten acciones específicas para lograr ese enfoque intercultural, ya que esto es lo que hace un currículo diferente a otras ofertadas por universidades con enfoque occidental.

4. Que la URACCAN Recinto Bilwi, contrate docentes de la especialidad con experiencia en docencia o que las autoridades académicas incluyan en la planificación anual cursos de especialización o postgrados, y de forma sistemática actualización en aspectos pedagógicos. 
5. La URACCAN a través de su vicerrector y coordinadora de carrera, a corto plazo revise, diseñe y ejecute el plan de investigación, según políticas de investigación y líneas de investigación de la carrera en el que participen más docentes y estudiantes.

6. Con fondos propios o proyectos, la administradora y vicerrector del recinto Bilwi garanticen la compra de materiales didácticos y requerimientos básicos, tales como: software con licencia y software libre, equipos, herramientas, adquisición y aseguramiento del recurso bibliográfico impreso y digital o bien una biblioteca virtual, según solicitud específica de la coordinación de carrera.

7. Que la secretaria académica y coordinadora de carrera proporcione sugerencias a la URACCAN Recinto Bilwi, que aseguren la iluminación adecuada, pupitres, ventilación apropiada según parámetros de higiene escolar.

8. La URACCAN a través de su vicerrector y coordinadora de carrera firmen convenios con universidades, instituciones estatales y privadas a nivel interinstitucional y firmar acuerdos con unidades académicas similares a nivel intrainstitucional para que la carrera de Informática Administrativa potencialice las prácticas y pasantías de docentes y estudiantes.

\section{Lista de referencias}

Caldera, T. M. (2007). Informe de seguimiento académico carrera licenciatura en Informática Administrativa.

Cunningham, M. (2002). La educación superior intercultural: Recurso pedagógico para una educación equitativa. BID.

Díaz, F; Arceo, B; Lule, M., Pacheco, D., Saad E. \& Rojas, S (2007). Metodología de diseño curricular para educación superior. México.

Quesada S. M. E, et al. (2001). El diseño curricular en los planes de estudio: aspectos teóricos y guía metodológica. San José Costa Rica: Editorial Universidad Nacional.

Roncal, F. (2004). Presentación Tendencias actuales de los currículos

REDICA/CSUCA/BID (2002). Manual del Sistema Centroamericano de Evaluación y Acreditación de Programas de Ingeniería.

Sáez, R. (2001). Ponencia: Tendencias actuales de la educación intercultural, presentada en las "Jornadas sobre Interculturalidad: Educar en las Aulas. Retos del siglo XXI", celebradas en Murcia.

Stufflebeam, D. I. (1971). Evaluación educativa. Itasca Illinois. 
Tünnermann, C. B. (2009). Nuevas perspectivas de la pertinencia y calidad de la Educación Superior. Accesado el 22 de septiembre, 2009 de http://www.elnuevodiario. com.ni/opinion/55553

Tünnermann, C. B. El Rol del Docente en la Educación Superior del Siglo XXI.

URACCAN (2004). Currículum de la Carrera de Licenciatura en Informática Administrativa.

URACCAN (2004). Modelo Pedagógico.

URACCAN (2008). Plan estratégico 2008 - 2012.

URACCAN (2005). Politica institucional de investigación. 